$\underline{\text { Preprint typeset in JHEP style. - HYPER VERSION }}$

BUHEP-02-24

$\mathrm{UW} / \mathrm{PT}-01 / 09$

HUTP-02/A016

\title{
The Minimal Moose for a Little Higgs
}

\author{
N. Arkani-Hamed \\ Jefferson Laboratory of Physics, Harvard University, Cambridge, MA 02138 \\ email: arkani@carnot.harvard.edu
}

A.G. Cohen

Physics Department, Boston University, Boston, MA 02215

email: cohen@bu.edu

E. Katz, A.E. Nelson

Department of Physics, Box 1560, University of Washington, Seattle, WA 98195-1560

email: amikatz@fermi.phys.washington.edu, anelson@phys.washington.edu

\section{T. Gregoire, J. Wacker}

Department of Physics, UC Berkeley, Berkeley, CA 94720, USA

email: gregoirt@socrates.berkeley.edu, jgwacker@socrates.berkeley.edu

\begin{abstract}
Recently a new class of theories of electroweak symmetry breaking have been constructed. These models, based on deconstruction and the physics of theory space, provide the first alternative to weak-scale supersymmetry with naturally light Higgs fields and perturbative new physics at the $\mathrm{TeV}$ scale. The Higgs is light because it is a pseudo-Goldstone boson, and the quadratically divergent contributions to the Higgs mass are cancelled by new $\mathrm{TeV}$ scale "partners" of the same statistics. In this paper we present the minimal theory space model of electroweak symmetry breaking, with two sites and four link fields, and the minimal set of fermions. There are very few parameters and degrees of freedom beyond the Standard Model. Below a TeV, we have the Standard Model with two light Higgs doublets, and an additional complex scalar weak triplet and singlet. At the $\mathrm{TeV}$ scale, the new particles that cancel the 1-loop quadratic divergences in the Higgs mass are revealed. The entire Higgs potential needed for electroweak symmetry breaking - the quartic couplings as well as the familiar negative mass squared - can be generated by the top Yukawa coupling, providing a novel link between the physics of flavor and electroweak symmetry breaking.
\end{abstract}




\section{Introduction}

The Standard Model provides an excellent description of all particle physics experiments performed to date. The parameterization of electroweak symmetry breaking in terms of a fundamental scalar field, however, is almost certainly incomplete. The quadratically divergent radiative corrections to the Higgs mass suggest a TeV-scale cutoff. A more complete, natural theory of electroweak symmetry breaking would include a stabilization mechanism for the Higgs mass through new physics at or below the TeV scale.

Precision electroweak measurements are consistent with perturbative standard model calculations and a light Higgs. The unreasonable effectiveness of this fundamental Higgs theory of electroweak symmetry breaking suggests that any new $\mathrm{TeV}$ scale physics that stabilizes the Higgs mass is also perturbative. A widely held belief is that the only perturbative candidate for electroweak symmetry breaking that stabilizes the weak scale is low-energy supersymmetry. In supersymmetric theories every Standard Model field has a superpartner of opposite statistics. The quadratic sensitivity of the Higgs mass to the TeV scale is removed by cancellations of the radiative corrections of Standard Model fields with those of their superpartners.

Recently, counter to this belief, a different class of models with perturbative physics stabilizing the electroweak scale has been introduced [1,2], based on "deconstruction" [3,4] and the physics of theory space $[3,5]$. In these models the light Higgs field appears as a pseudo-Goldstone boson at $\mathrm{TeV}$ energies and below, with conventional gauge, Yukawa and self-couplings. The quadratic sensitivity to the cutoff scale that these couplings normally induce are cancelled, not by particles of opposite statistics, but instead by particles of the same statistics. Global symmetries of the theory ensure these cancellations. The interactions responsible for the symmetry breaking giving rise to the pseudo-Goldstone bosons are characterized by a scale $\sim 10 \mathrm{TeV}$.

The models described in [1] are non-linear sigma models characterized by a toroidal "theory space". The small example described in detail in [2] has 4 sites (corresponding to a gauge group $S U(3)^{3} \times S U(2) \times U(1)$ ) and 8 links (corresponding to 8 sets of non-linear sigma model fields). Some of these sigma model fields are eaten, higgsing the gauge group to the Standard Model group $S U(2) \times U(1)$ and giving $\mathrm{TeV}$ scale masses to the corresponding gauge bosons. Additional operators break the global symmetries and give $\mathrm{TeV}$ scale masses to most of the scalars, leaving two pseudo-Goldstone multiplets massless at tree level. These include a pair of Standard Model Higgs doublets, interacting through a quartic potential. A set of colored, vector-like fermions were introduced to generate the top quark Yukawa coupling. The additional TeV scale degrees of freedom cancel the one-loop Standard Model quadratic divergence in the Higgs mass: the massive gauge bosons cancel the divergence from the Standard Model gauge loop; the massive scalars cancel the divergence from the Higgs self-coupling; the massive fermions cancel the divergence from the top quark loop. Such models are consistent with precision electroweak constraints (such as the $S$-parameter), and flavor model building in this context can be explored relatively unfettered by the bonds of flavor violation. 
As emphasized in [1], the essential idea of the Higgs as a pseudo-Goldstone boson and the models constructed are independent of extra dimensions and their deconstruction. Models based on deconstruction have the virtue of a large collection of approximate symmetries protecting the Higgs mass, and a restricted set of symmetry breaking effects. The symmetry and field content of these theories are naturally represented graphically as sites and links, in a notation sometimes referred to as "moose" [6] or "quiver" [7]. This construction makes it evident that these theories realize a theory space in which symmetry breaking terms are localized, while the lightest pseudo-Goldstone bosons are extended objects: non-contractible loops in theory space. Such extremely light Goldstone bosons, which can only receive mass from the combined efforts of more than one symmetry breaking term, are known as "little Higgses". A general analysis [8] reveals that little Higgses are associated with topological properties of theory spaces: each little Higgs corresponds to an element of the fundamental group of the theory space; and the little Higgs potential is obtained from the group relations.

In this paper and its companion [9] we further abstract the essential features of the little Higgs idea. We construct models, based on a moose with 2 sites and 4 links, which have the same low-energy structure as the toroidal theory space models, and are economical enough to obviate the need for moose notation. We also point out a new possibility for the origin of the Higgs potential: both the Higgs negative mass squared and the quartic couplings can have their source in the same operators that generate the top Yukawa coupling. This leads to a relation between the Higgs and top masses $m_{H} \sim m_{t}$, and a fascinating link between the physics of flavor and electroweak symmetry breaking.

The companion paper [9] considers sigma models based on more general cosets, with no obvious moose description. Remarkably the Higgs quartic potential in such models can be generated by the gauge interactions alone. These more general coset constructions allow the theory below a $\mathrm{TeV}$ to contain only the Standard Model particles with a single Higgs, and the smallest number of new states at a TeV.

\section{A Minimal Moose}

\subsection{The Model}

As a warm-up, in this section we present an example of a minimal moose with only two sites and four links. It is related to the $2 \times 2$ toroidal construction of [1], but is half as large ${ }^{1}$.

The gauge symmetry at one of the sites is $G_{1}=S U(3)$. (Alternatively an $S U(2) \times U(1)$ subgroup would suffice.) At the other site the gauge symmetry is $G_{2}=S U(2) \times U(1)$. There are 4 link fields $X_{j}, j=1, \cdots, 4$, which are $3 \times 3$ non-linear sigma model fields $X_{j}=\exp \left(2 i x_{j} / f\right)$, each transforming as a bi-fundamental under $G_{1} \times G_{2}$, where a fundamental of $G_{2}$ is taken to be $\mathbf{2}_{1 / 6} \oplus \mathbf{1}_{-1 / 3}$. All the Standard Model fermions are charged under the $G_{2}$ gauge symmetry with their usual quantum numbers, so the model is anomaly free. The

\footnotetext{
${ }^{1}$ To see the relation, consider a version of the 4 site toroidal moose with $2 S U(2) \times U(1)$ sites and $2 S U(3)$ sites - the $S U(2) \times U(1)$ sites are at opposite corners. Now identify links and sites which are exchanged by a $Z_{2}$ discrete symmetry, or, in other words, orbifold by a translation along the diagonal of the moose.
} 
theory has a large, approximate $S U(3)^{8}$ global symmetry spontaneously broken to $S U(3)^{4}$, with the non-linear realization

$$
X_{j} \rightarrow L_{j} X_{j} R_{j}^{\dagger}, \quad j=1,2,3,4 .
$$

The cutoff of this non-linear sigma model is $\Lambda \sim 4 \pi f$, which we take to be $\sim 10 \mathrm{TeV}$. The effective theory beneath this cutoff is described by the Lagrangian

$$
\mathcal{L}=\mathcal{L}_{G}+\mathcal{L}_{X}+\mathcal{L}_{t}+\mathcal{L}_{\psi}
$$

Here $\mathcal{L}_{G}$ includes the conventional non-linear sigma model field kinetic terms and gauge interactions, while $\mathcal{L}_{X}$ contains "plaquette" couplings between the $X_{j}$ :

$$
\mathcal{L}_{X}=f^{4} \operatorname{tr}\left(A X_{1} X_{2}^{\dagger} X_{3} X_{4}^{\dagger}\right)+f^{4} \operatorname{tr}\left(A^{\prime} X_{1} X_{4}^{\dagger} X_{3} X_{2}^{\dagger}\right)+\text { h.c. }
$$

where $A=\kappa \mathbf{1}+\epsilon \mathbf{T}_{8}$ and $A^{\prime}=\kappa^{\prime} \mathbf{1}+\epsilon^{\prime} \mathbf{T}_{8}$. Each of these terms breaks the global symmetries, but preserves a sufficiently large subgroup of the global symmetries to leave some Goldstone bosons massless. In writing down these couplings, we allow a specific set of global symmetry breaking operators without addressing their UV origin. We then include all terms needed to renormalize the theory at 1-loop, with coefficients which are no smaller than their natural size. Possible difficulties with a natural origin of the plaquette terms from QCD-like UV completions of the non-linear sigma model are discussed in ref. [10]. In section 3, we show that the necessary plaquette terms can be generated, quite naturally, from the top sector.

The remaining terms generate the Standard Model Yukawa couplings; to avoid introducing quadratic divergences, we also introduce a vector-like pair of colored Weyl fermions $U, U^{c}$ and couple them to the top quark with $\mathcal{L}_{t}$ :

$$
\mathcal{L}_{t}=\lambda f\left(\begin{array}{lll}
0 & 0 & u_{3}^{c \prime}
\end{array}\right) X_{1} X_{2}^{\dagger}\left(\begin{array}{l}
q_{3} \\
U
\end{array}\right)+\lambda^{\prime} f U U^{c} .
$$

Finally, $\mathcal{L}_{\psi}$ contains the Yukawa couplings for the light fermions; since these are very small, the quadratic divergences associated with them are negligible for our cutoff $\Lambda \sim 10 \mathrm{TeV}$. For the light up-type quarks, $\mathcal{L}_{\psi}$ has the same form as $\mathcal{L}_{t}$ with $U, U^{c}$ removed, while for the down and charged lepton sectors $\mathcal{L}_{\psi}$ contains

$$
\mathcal{L}_{\psi} \supset\left(\begin{array}{ll}
q & 0
\end{array}\right) X_{1} X_{2}^{\dagger} \lambda_{\mathrm{D}} f\left(\begin{array}{c}
0 \\
0 \\
d^{c}
\end{array}\right)+\left(\begin{array}{ll}
l & 0
\end{array}\right) X_{1} X_{2}^{\dagger} \lambda_{\mathrm{E}} f\left(\begin{array}{c}
0 \\
0 \\
e^{c}
\end{array}\right) .
$$

This completes the description of the model.

\subsection{Tree-level spectrum and interactions}

Let us examine this theory at tree-level, for the moment putting $\epsilon=\epsilon^{\prime}=0$ in $\mathcal{L}_{X}$. The nonlinear sigma model fields Higgs the $G_{1} \times G_{2}$ gauge group down to the $S U(2) \times U(1)$ subgroup. 
The massive $S U(3)$ gauge bosons have a mass $\sim g f$. The linear combination of Goldstone bosons $\left(x_{1}+\cdots x_{4}\right)$ is eaten, while the quartic terms in $\mathcal{L}_{X}$ give mass to the linear combination $x_{1}-x_{2}+x_{3}-x_{4}$, of order $\sim \kappa f$. (Here and in what follows we assume $\kappa, \kappa^{\prime}$ are real and positive, although it suffices for $\Re\left(\kappa, \kappa^{\prime}\right)>0$.) At tree-level two orthogonal combinations of pseudo-Goldstone boson multiplets, which we can take to be $x_{1}-x_{3}$ and $x_{2}-x_{4}$, are massless. They decompose under the unbroken $S U(2) \times U(1)$ gauge symmetry as a pair of Higgs doublets, as well as a complex weak triplet and singlet. Furthermore, these classically massless pseudo-Goldstone bosons, or "little Higgses", receive no 1-loop quadratically cutoff sensitive corrections to their masses.

At tree-level the little Higgses interact through a quartic potential, with one linear combination of the doublets coupling to the top quark. The quartic potential can very quicly be found in the limit $\kappa=\kappa^{\prime}$; with this choice $\mathcal{L}_{X}$ has a symmetry under which $X_{j} \rightarrow X_{j}^{\dagger}$, or equivalently $x_{j} \rightarrow-x_{j}$. This ensures that the potential is even in the fields, and in deriving the potential for the light fields, we can simply set the heavy fields to zero. The light fields corresponding to $x_{1}-x_{3}$ and $x_{2}-x_{4}$ can be parameterized as

$$
\begin{aligned}
& X_{1}=X_{3}^{\dagger}=U \equiv e^{2 i(x+y) / f} \\
& X_{4}=X_{2}^{\dagger}=V \equiv e^{2 i(x-y) / f} .
\end{aligned}
$$

The plaquette interactions then give rise to the potential

$$
-\kappa f^{4} \operatorname{tr}\left(U V U^{-1} V^{-1}\right)+V \leftrightarrow V^{-1}+\text { h.c. }=\kappa \operatorname{tr}[x, y]^{2}+\cdots
$$

where we have furhter assumed for simplicity that $\kappa$ is real.

It is extremely interesting that we have found a potential for $x, y$ with no mass terms but with a quartic potential. Why did this happen? In order to understand this, first take a limit where one of the plaquettes is turned off, say by taking $\kappa^{\prime}=0$. The $\kappa$ plaquette still gives mass to the linear combination $x_{1}-x_{2}+x_{3}-x_{4}$, but it is easy to see that no potential can be generated at all for the other goldstones. This is because the $\kappa$ plaquette still preserves an $S U(3)^{4}$ subgroup of the $S U(3)^{8}$ global symmetry, where $R_{1}=R_{2}, L_{2}=L_{3}, R_{3}=R_{4}, L_{4}=L_{1}$. This is spontaneously broken to the diagonal $S U(3)$, leaving three exactly massless goldstone bosons (one of which is eaten). We can also see this directly in expanding the plaquette interaction in terms of the heavy multiplet $z \propto x_{1}-x_{2}+x_{3}-x_{4}$ as well as the uneaten $x, y$ fields, which yields schematically

$$
\kappa \operatorname{tr}(f z+i[x, y]+\cdots)^{2}
$$

Upon integrating out the heavy $z$ multiplet there is no potential for $x, y$. Diagramatically, there is a quartic coupling $\operatorname{tr}[x, y]^{2}$, as well as a cubic coupling $\operatorname{tr} z[x, y]$. Integrating out $z$ exactly cancels the quartic coupling, as it must since $x, y$ are exact Goldstone bosons. An exactly analagous argument holds for the $\kappa^{\prime}$ coupling. However, $\kappa^{\prime}$ preserves a different $S U(3)^{4}$ global symmetry, and the potential from the $\kappa^{\prime}$ plaquette is of the form

$$
\kappa^{\prime} \operatorname{tr}(f z-i[x, y]+\cdots)^{2}
$$


In the presence of both $\kappa$ and $\kappa^{\prime}$, there is only an $S U(3) \times S U(3)$ global symmetry broken to $S U(3)$, and only one exactly massless goldstone boson, which is the one that is eaten. Therefore, $x, y$ are not exact Goldstone bosons and can acquire a potential. However, since their potential is the sum of two pieces, one proportional to $\kappa$ and the other proprtional to $\kappa^{\prime}$, it is impossible for $x, y$ to pick up a mass at tree level. They can acquire a quartic potential, however: upon integrating out $z$ we have

$$
\frac{\kappa \kappa^{\prime}}{\kappa+\kappa^{\prime}} \operatorname{tr}[x, y]^{2}
$$

Note the non-analytic dependence on $\kappa, \kappa^{\prime}$ in the denominator, arising from integrating out $z$ which has a mass squared proprtional to $f^{2}\left(\kappa+\kappa^{\prime}\right)$. This quartic coupling vanishes as it must in the limit where either $\kappa$ or $\kappa^{\prime}$ vanishes. Thus we have generated a quartic potential for the little Higgses, without a mass term, by breaking the global symmetry with two different couplings. Any one of these couplings preserves enough global symmetry to ensure that the little Higgses are exact Goldstone bosons. But together the couplings break all these symmetries and the little Higgses can acquire a quartic potential. In the next section, we see that this same mechanism ensures the absence of 1-loop quadratic divergences for the mass of the little Higgses.

We can exhibit the components of $x$ as a $3 \times 3$ hermitian matrix

$$
x=\left(\begin{array}{cc}
\varphi_{x}+\eta_{x} & h_{x} \\
h_{x}^{\dagger} & -2 \eta_{x}
\end{array}\right)
$$

and similarly for $y$. Here $\varphi, \eta$ are fields in the $\mathbf{3}_{0}, \mathbf{1}_{0}$ representation of $S U(2) \times U(1)$ respectively, while the $h$ have the quantum numbers $\mathbf{2}_{1 / 2}$ of the standard model Higgs. The quartic potential is then

$$
\kappa \operatorname{tr}[x, y]^{2}=\kappa \operatorname{tr}\left(h_{x} h_{y}^{\dagger}-h_{y} h_{x}^{\dagger}\right)^{2}+\kappa\left(h_{x}^{\dagger} h_{y}-h_{y}^{\dagger} h_{x}\right)^{2}+\text { terms involving } \varphi, \eta .
$$

This can be recast in a more familiar form by defining $h_{1}=h_{x}+i h_{y}, h_{2}=h_{x}-i h_{y}$. The quartic potential is then

$$
\kappa \operatorname{tr}\left(h_{1} h_{1}^{\dagger}-h_{2} h_{2}^{\dagger}\right)^{2}+\kappa\left(h_{1}^{\dagger} h_{1}-h_{2}^{\dagger} h_{2}\right)^{2}+\text { terms involving } \varphi, \eta .
$$

This two-Higgs doublet quartic potential is similar to that of the supersymmetric Standard Model.

To exhibit the top Yukawa coupling, we expand $\mathcal{L}_{t}$ to first order in the Higgs doublet fields

$$
\lambda u_{3}^{c \prime}\left(f U+h_{x} q_{3}\right)+\cdots+\lambda^{\prime} f U U^{c}
$$

One linear combination of $U^{c}$ and $u_{3}^{c \prime}$ marries $U$ to become a massive fermion with mass $\sim \lambda f$; the orthogonal combination $u_{3}^{c}$ remains massless with a Yukawa coupling to $q_{3}$

$$
\lambda_{t} u_{3}^{c} h_{x} q_{3}, \quad \text { where } \quad \lambda_{t}=\frac{\lambda \lambda^{\prime}}{\sqrt{\lambda^{2}+\lambda^{\prime 2}}}
$$


The mixing of the top quark with vector-like Fermions at the TeV scale is similar to FrogattNielsen models of flavor [11] and the top see-saw [12,13].

In summary, at the classical level there are two massless Higgs doublets, together with a complex triplet and singlet. These scalars have a tree-level quartic potential, and one linear combination of the Higgs doublets has a Yukawa coupling to the top quark fields $q_{3}, u_{3}^{c}$. We also have a set of massive vectors, scalars and fermions with masses $\sim g f, \kappa f, \lambda f$ respectively. All these scales are of order a TeV.

\subsection{Power-counting and absence of 1-loop quadratic divergences}

Radiative corrections generate masses for the little Higgses that are only logarithmically sensitive to the cutoff. We establish this through two different routes. First we examine how the non-linearly realized symmetries which protect the little Higgs masses are explicitly broken: we show that any one of the gauge, plaquette or Yukawa interactions alone preserve enough of these symmetries to forbid masses for the Goldstone multiplets. Any quadratically divergent correction to the masses must then arise from a combination of more than one of these couplings, and is absent at 1-loop. Secondly we give a simple, general set of rules which are sufficient (though not necessary) to ensure an arbitrary theory space to be free of 1-loop quadratic divergences, and verify the validity of these rules by directly computing the 1-loop Coleman-Weinberg potential. Our model can trivially be seen to satisfy these rules.

The nonlinearly realized $S U(3)^{8}$ symmetry is explicitly broken by the gauge, plaquette and Yukawa interactions, and these will in turn induce other operators. We do a standard power-counting analysis [14-16] in order to determine the natural size of these interactions. This is most straightforwardly done following $[15,16]$. The Lagrangian is written as $\mathcal{L}=$ $\Lambda^{4} / 16 \pi^{2} \hat{\mathcal{L}}$, where $\Lambda \sim 4 \pi f$ is the UV cutoff and all the mass scales in $\hat{\mathcal{L}}$ are scaled by powers of $\Lambda$. This rule leads to the familar $f^{2}$ coefficient for the goldstone kinetic terms. Also all non-derivative terms involving the $X$ 's naturally have a coefficient $\Lambda^{2} f^{2}$, while the fermion mass terms and coupling to $X^{\prime} s$ are scaled by $\Lambda$. The small symmetry breaking effects of the spurions in $\mathcal{L}_{X}$ then have small dimensionless size $\sim \kappa^{(\prime)} /\left(16 \pi^{2}\right), \epsilon^{(\prime)} /(16 \pi)^{2}$, while those in $\mathcal{L}_{t}$ are $\sim \lambda^{(\prime)} /(4 \pi)$. Note that we can independently rephase $\lambda$ and $\lambda^{\prime}$ by rephasing the fermions $u^{c}$ and $U^{c}$, therefore in any induced operator only involving the $X$ 's, these spurions can only enter as $\left|\lambda^{(\prime)} / 4 \pi\right|^{2}$. The gauge interaction spurion is $g^{2} /\left(16 \pi^{2}\right)$. Therefore each one of our spurions counts as a loop factor.

The power-counting is now staightforward. Every operator is proportional $\Lambda^{2} f^{2}$ times the appropriate product of spurions needed to generate it. In particular, any induced little Higgs masses are proprtional to $\Lambda^{2}$ times the product of spurions. The generation of little Higgs masses will require at least 2 spurions, and therefore will have quadratic sensitivity to the cutoff only at 2-loop level. This happens because each one of our interaction terms preserves a large subset of the global symmetry. Consider the limit where only one of the gauge couplings, say the $G_{1}$ gauge coupling, is non-zero. This coupling preserves a symmetry under which $L_{1}=L_{2}=\cdots=l$ but all four of the $R_{j}$ arbitrary. One combination of the Goldstone multiplets is eaten, but three sets of Goldstone modes remain massless. Exactly the same 
happens when only the couplings of $G_{2}$ are non-zero. The presence of both couplings breaks all the chiral symmetries and there are no exact Goldstone modes. However two sets are left classically massless. Any quadratically divergent mass must involve both couplings, arising at 2-loop order. A similar analysis applies to the plaquette interactions. As we discussed in the last section, in the limit where only $\kappa$ is non-zero, the global symmetry is the $S U(3)^{4}$ subgroup of $S U(3)^{8}$ with $R_{1}=R_{2}, L_{2}=L_{3}, R_{3}=R_{4}, L_{4}=L_{1}$. This is spontaneously broken to the diagonal $S U(4)$, leaving three massless Goldstone multiplets. With only $\kappa^{\prime}$ non-zero a different $S U(3)^{4}$ is broken, while in the presence of both $\kappa$ and $\kappa^{\prime}$ only an $S U(3) \times S U(3)$ symmetry is left, with the one exact Goldstone boson eaten via the Higgs mechanism. The remaining two approximate Goldstone multiplets only acquire quadratically divergent masses if both $\kappa$ and $\kappa^{\prime}$ are present, again requiring at least 2 loops. Finally a similar analysis applies to the fermions. The addition of $U$ to the $q_{3}$ gives the $\lambda$ piece of $\mathcal{L}_{t}$ an $S U(3)$ global symmetry, which ensures that $h_{x}$ is a Goldstone mode. This symmetry is broken by the mass term $\lambda^{\prime} f U U^{c}$. But again this requires both $\lambda$ and $\lambda^{\prime}$, and arises only at 2-loop level. Note that it is important here that there are rephasing symmetries that force any one of our spurions to appear quadratically as $\left|\lambda^{(\prime)} /(4 \pi)\right|^{2}$. This would not be the case if we add a mass term $\lambda^{\prime \prime} f u_{3}^{c \prime} U$. Then, the combination $\left(\lambda^{*} / 4 \pi\right)\left(\lambda^{\prime \prime} / 4 \pi\right)$ is invariant, and would lead to a 1-loop quadratically divergent Higgs mass. However this $u_{3}^{c \prime} U$ mass term can be prohibited by chiral symmetries.

There are simple rules which are sufficient (although not necessary) to ensure the absence of 1-loop quadratic divergences for the little Higgs mass from the gauge, quartic and top Yukawa sectors in a general moose. For the gauge and quartic couplings these rules are simply phrased as properties of the theory space:

Gauge couplings: Every link connects two different sites.

Quartic couplings: No plaquette contains the same link twice.

There are a variety of ways to ensure the absence of 1-loop quadratic divergences from the top sector. The simplest possibility is just what we have done for $\mathcal{L}_{t}$, but which we phrase here slightly more generally:

Top Yukawa couplings: The top Yukawa comes from interactions of the form

$$
\lambda f\left(\begin{array}{lll}
0 & 0 & u_{3}^{c \prime}
\end{array}\right) W\left(\begin{array}{l}
q_{3} \\
U
\end{array}\right)+\lambda^{\prime} f U U^{c}
$$

where $W$ is a product of link fields.

Note that our specific 2-site, 4-link theory space satisfies all of these properties: each link connects the two sites, the plaquette interactions in $\mathcal{L}_{X}$ contain each link only once, and $\mathcal{L}_{t}$ has precisely the form of our rule. This also makes it clear why the minimal model has 2 sites and 4 links. These rules are a manifestation of the general requirement that all order one symmetry breaking terms must preserve at least one global symmetry under which the 
little Higgses transform nonlinearly. Any order one couplings for the little Higgses must arise from a collaboration between at least two such symmetry breaking terms.

To show that these rules suffice to eliminate 1-loop quadratic divergences to the little Higgs masses, we compute the quadratically divergent part of the 1-loop Coleman-Weinberg potential. We turn on a background $X_{i}=\tilde{X}_{i}$ for the link fields. The 1-loop quadratic divergences in the Coleman-Weinberg potential are proportional to

$$
\frac{\Lambda^{2}}{16 \pi^{2}} \operatorname{Str} M^{\dagger} M[\tilde{X}]
$$

We must therefore calculate $\operatorname{Str} M^{\dagger} M[\tilde{X}]$ where $M[\tilde{X}]$ is the mass matrix of the theory in the presence of the background.

First consider the gauge sector. Consider a link field between two different sites $i$ and $j$. The gauge boson mass matrix comes from expanding the covariant kinetic term for the link fields to quadratic order in the gauge fields, yielding $A_{i}^{a}\left(M^{2}[\tilde{X}]\right)_{a b}^{i j} A_{j}^{b}$, where $a, b$ are gauge indices and

$$
M_{a b}^{2}[\tilde{X}]=\frac{f^{2}}{4}\left(\begin{array}{cc}
\frac{1}{2} g_{i}^{2} \delta_{a b} & g_{i} g_{j} m_{a b}^{2} \\
g_{i} g_{j} m_{a b}^{2 \dagger} & \frac{1}{2} g_{j}^{2} \delta_{a b}
\end{array}\right) \quad m_{a b}^{2}=\operatorname{tr} \mathbf{T}_{a} \tilde{X} \mathbf{T}_{b} \tilde{X}^{\dagger}
$$

The important point is that $M_{a a}^{2}$ is always independent of the background field, $\tilde{X}$, and therefore so is the trace. Hence there are no 1-loop gauge quadratic divergences for any link field mass. This argument breaks down if a link field connects a site to itself.

Now consider the 1-loop corrections involving the quartic couplings, which arise from the plaquette interactions. Consider a general plaquette:

$$
V\left(X_{i}\right)=-\kappa f^{4} \operatorname{tr} M_{1} X_{1} \cdots M_{N} X_{N}+\text { h.c. }
$$

where the $M_{i}$ are arbitrary matrices. Write the link fields as a linearized fluctuation, $x_{i} \equiv$ $x_{i}^{a} \mathbf{T}_{a}$, about a background field, $\tilde{X}_{i}: X_{i}=\exp \left(i x_{i}\right) \tilde{X}_{i}$. In this decomposition, the kinetic terms for $x_{i}^{a}$ are independent of the background field, which greatly simplifies the analysis. We expand the plaquette to quadratic order in the fluctuations and find the mass matrix, $x_{i}^{a}\left(M^{2}\right)_{a b}^{i j} x_{j}^{b}$. The diagonal component of this mass matrix is

$$
\left(M^{2}\right)_{a b}^{i i} \sim \kappa f^{2} \operatorname{tr} M_{1} \tilde{X}_{1} \cdots M_{i} \mathbf{T}_{a} \mathbf{T}_{a} \tilde{X}_{i} M_{i+1} \tilde{X}_{i+1} \cdots M_{N} \tilde{X}_{N}+\text { h.c. }
$$

Summing over the diagonal entries of the mass matrix and using $\sum_{a} \mathbf{T}_{a}^{2} \propto \mathbf{1}$, we find $\operatorname{tr} M^{2} \propto \operatorname{tr} M_{1} \tilde{X}_{1} \cdots M_{i} \tilde{X}_{i} M_{i+1} \tilde{X}_{i+1} \cdots M_{N} \tilde{X}_{N}+$ h.c., which is just the plaquette operator itself! Therefore, the 1-loop quadratic divergences only renormalize the plaquette interactions, and do not generate any new operators in the theory. If a field appears in a plaquette more than once, then this argument breaks down: the mass matrix will have a more complicated form with $X$-dependent diagonal entries.

Let us finally check that the absence of 1-loop quadratic divergences from the Yukawa couplings. For this purpose, it is enough to consider only the interaction proportional to $\lambda$. 
The mass matrix for the relevant fermions in a general background $\tilde{W}$ is then

$$
M=\lambda f P \tilde{W}, \text { where } P=\operatorname{diag}(0,0,1) .
$$

The quadratic divergence is then proportional to

$$
\operatorname{tr} M^{\dagger} M=\lambda^{2} f^{2} \operatorname{tr} \tilde{W}^{\dagger} P P \tilde{W}=\lambda^{2} f^{2} \operatorname{tr} P
$$

which is independent of the background $\tilde{W}$. Once again, there are no 1-loop quadratic divergences. Note that the presence of the $U$ fields is crucial here. In its absence, we would have instead $M=\lambda f P \tilde{W}(1-P)$, and $\operatorname{tr} M^{\dagger} M$ would indeed depend on the background field $\tilde{W}$. This is of course a direct consequence of our spurion analysis. In the absence of $\lambda^{\prime}$, the Yukawa sector has an enhanced $S U(3)$ global symmetry acting on $W$ and the $\left(q_{3}, U\right)$ triplet, and no potential for the components of $W$ can be generated.

We have verified the absence of quadratic divergences explicitly by seeing that the trace of the mass squared matrix for the fields in the theory is independent of the background little Higgs fields. In terms of mass eigenstates, this means that as the little Higgs background is turned on, the classically massless modes become heavier, but the heavy modes become lighter in just such a way that the sum of the mass squareds is independent of the background. Thus the cancellation of quadratic divergences is between particles of the same statistics: the massive gauge bosons cancel the quadratic divergences associated with the massless Standard Model gauge fields, the massive scalars cancel the quadratic divergence associated with the little Higgs quartic coupling, and the massive fermion cancels the quadratic divergence from the top-Yukawa coupling.

\subsection{Electroweak symmetry breaking}

While there are no quadratically divergent corrections to the masses of the little Higgses, in this model there are logarithmically divergent corrections at 1-loop, and quadratically divergent 2-loop corrections. In the general model of [1], the quadratic divergences can be pushed to $N+1$ loops. However, there are always finite 1-loop corrections to the Higgs mass squared, which are at least of order $3 \lambda_{t}^{4} f^{2} /\left(16 \pi^{2}\right)$ and so we expect $f \sim \mathrm{TeV}$. Since the cutoff can not go far above $4 \pi f \sim 10 \mathrm{TeV}$, removing quadratic divergences beyond 1-loop is an unnecessary extravagance. In order to compute the magnitude and sign of the radiative corrections to the little Higgs masses in our model, we look at the 1-loop contribution, which is slightly log enhanced.

The gauge and quartic couplings give a positive mass squared to all of $\phi_{x, y}, \eta_{x, y}, h_{x, y}$. It is easy to understand this qualitatively: the usual quadratic divergences of the low energy theory are cut off at the mass of the heavy field which cancels the divergence. For instance, the gauge loops generate a mass squared $\sim\left[g^{2} /\left(16 \pi^{2}\right)\right](g f)^{2}$. The fermion loop does not generate

any potential for $\phi_{x, y}, \eta_{x, y}$, since the interactions in $\mathcal{L}_{t}$ do not break the $S U(2) \times U(1)$ chiral symmetries under which the $\phi$ and $\eta$ fields transform nonlinearly. However, the fermion loop does produce a negative mass squared $\sim-\left[3 \lambda^{2} /\left(16 \pi^{2}\right)\right](\lambda f)^{2}$ for $h_{x}$, which can dominate over 
the positive gauge and plaquette contributions, and so we can have $m_{h_{y}}^{2}>0$ while $m_{h_{x}}^{2}<0$. This forces $h_{x}$ to acquire a vev. However the quartic potential has flat directions where $h_{x}$ has any value while $h_{y}=0$, and therefore $h_{x}$ runs away along this flat direction. The flat direction analysis is perhaps more familiar in the $h_{1}, h_{2}$ basis, where our quartic potential has the same form as in the supersymmetric standard model. In this basis, the Higgs mass terms are of the form $\left(m_{x}^{2}+m_{y}^{2}\right)\left(\left|h_{1}\right|^{2}+\left|h_{2}\right|^{2}\right)+\left(m_{x}^{2}-m_{y}^{2}\right) h_{1}^{\dagger} h_{2}+$ h.c. The familiar flat direction where $h_{1}=h_{2}$ is not stabilized since $m_{h_{1}}^{2}=m_{h_{2}}^{2}$. This is why we have added the $\epsilon, \epsilon^{\prime}$ terms to $\mathcal{L}_{X}$, where $\epsilon$ can be naturally small. Expanding these pieces to quadratic order generates the mass term $\Im\left(\epsilon^{\prime}-\epsilon\right) f^{2}\left(\left|h_{1}\right|^{2}-\left|h_{2}\right|^{2}\right)$, which splits $h_{1}, h_{2}$ and lifts the flat direction, stabilizing electroweak symmetry breaking.

We have seen that EWSB can arise naturally in this very simple model with a light Higgs, avoiding 1-loop quadratic divergences, and allowing for a cutoff $\Lambda \sim 10 \mathrm{TeV}$.

\subsection{Precision Electroweak and FCNC constraints}

The bounds from precision electroweak data can be satisfied, since all the new physics in these models decouples in the large mass limit. There are decoupling effects that can nevertheless be close to experimental bounds. For instance, the expansion of the non-linear sigma model kinetic terms can give rise to operators that violate custodial $S U(2)$ and generate a contribution to the $\rho$ (or $T$ ) parameter. Also, the triplet fields $\phi$ can acquire vacuum expectation values, after electroweak symmetry breaking, from cubic terms of the form $h \phi h$, and contribute to the $T$ parameter. Such couplings may arise at tree-level for the heavy triplets, or at 1-loop for the light triplets. However, all of these correction to $m_{W} / m_{Z}$ are of order $\sim(v / f)^{2}$, parametrically the same size as 1-loop Standard Model corrections, and give a correction to $T$ close to the bound. Another potential problem is that a cutoff-sensitive tadpole might be generated for the light singlets, which would lead to a singlet vev of order $f$ and destabilize the desired ground state. In [2] a $Z_{4}$ global symmetry was imposed which forbids such a tadpole and also makes this singlet a stable dark matter candidate. However the $Z_{4}$ symmetry is not necessary simply to eliminate the tadpole. We have seen directly that no tadpole is generated at 1-loop: the plaquette interactions do not contain linear terms, and the fermion loop does not generate any potential for the triplets or singlets. More generally, the generation of any such tadpole requires both interactions which break the global diagonal $S U(3)$ and interactions which break the nonlinearly realized $U(1)$ subgroups of the $S U(3)^{8}$ under which the $\eta^{\prime}$ s transform nonlinearly. The $\kappa, \kappa^{\prime}$ terms in $\mathcal{L}_{X}$ preserve the $S U(3)$, while $\mathcal{L}_{G}$ or $\mathcal{L}_{t}$ preserve all the $U(1)^{\prime}$ s. Any tadpole must involve at least $\kappa, \kappa^{\prime}$ and one of the other couplings, and is small enough that any resulting singlet vev is of order $M_{W}$ or less. An $\eta$ tadpole is also forbidden by $C P$, since the $\eta$ 's are $C P$-odd. Therefore, if $\kappa, \kappa^{\prime}$ are real, no $\eta$ tadpole is ever generated from this sector of the theory. The $\epsilon, \epsilon^{\prime}$ terms break both the $S U(3)$ and some of $U(1)$ 's, as well as $C P$. Any tadpole involving one of these couplings must further involve at least a $\kappa$ or $\kappa^{\prime}$, again to leading to an acceptably small tadpole.

Another challenge for TeV extensions of the Standard Model is ensuring sufficient suppression of flavor changing neutral currents (FCNC). Any flavor changing neutral currents 
mediated by particles in this effective theory are smaller than those of the Standard Model. Furthermore, our cutoff is high enough to make most FCNC from physics above the cutoff sufficiently small. There are a few significant constraints on flavor physics and the UV origin of $\mathcal{L}_{\psi}$, particularly from kaon $C P$ violation and $D-\bar{D}$ mixing [17]. As an existence proof that FCNC are not necessarily a problem, we note that this moose is easily UV completed into a renormalizable supersymmetric theory with supersymmetry breaking scale of order the cutoff [18] which allows the couplings needed for quark and lepton masses while satisfying FCNC constraints. An alternative is a "cascade" theory, in which the nonlinear sigma model is UV completed into a linear sigma model whose sigma field is itself a little Higgs of a nonlinear sigma model with a cutoff on the order of hundreds of $\mathrm{TeV}$ - high enough that FCNC from beyond are not a problem.

\section{Higgs potential from Top Yukawa}

In this section, we construct a model where all of the interactions needed to generate the Higgs potential - both the negative mass squared and the plaquette interactions producing the quartic interactions - arise from the same couplings that generate the top Yukawa coupling. The site and link structure is exactly the same as before. We add two sets of vector-like triplet Fermions to the theory, $T_{1,2}, T_{1,2}^{c}$, where $T_{i}=\left(Q_{i}, U_{i}\right), T_{i}^{c}=\left(Q_{i}^{c}, U_{i}^{c}\right)$. The Lagrangian is of the form

$$
\mathcal{L}=\mathcal{L}_{G}+\mathcal{L}_{\psi}+\mathcal{L}_{t}^{\prime}+\cdots
$$

Note that we don't have any "plaquette" interactions analagous to $\mathcal{L}_{X}$; neverthelss, we will see that these interactions must be included with the naturally correct size, induced from those in the Yukawa interactions of $\mathcal{L}_{t}^{\prime}$. $\mathcal{L}_{t}^{\prime}$ is of the form

$$
\mathcal{L}_{t}^{\prime}=\mathcal{L}_{T}+\mathcal{L}_{m i x}
$$

Here $\mathcal{L}_{T}$ represents the interaction of the vector-like $T, T^{c}$ with the $X^{\prime} s$ as

$$
\mathcal{L}_{t}^{\prime}=f T_{1}^{c} W_{1} T_{1}+f T_{2}^{c} W_{2} T_{2}
$$

where

$$
W_{1}=\lambda_{1} X_{2} X_{1}^{\dagger}+\tilde{\lambda}_{1} X_{3} X_{4}^{\dagger}, \quad W_{2}=\lambda_{2} X_{4} X_{1}^{\dagger}+\tilde{\lambda}_{2} X_{3} X_{2}^{\dagger}
$$

while $\mathcal{L}_{\text {mix }}$ are mass mixing terms between $T, T^{c}$ and the Standard Model top sector $q_{3}^{\prime}, u_{3}^{c \prime}$

$$
\mathcal{L}_{\text {mix }}=f q_{3}^{\prime}\left(\zeta_{1} Q_{1}^{c}+\zeta_{2} Q_{2}^{c}\right)+f u_{3}^{c \prime}\left(\tilde{\zeta}_{1} U_{1}+\tilde{\zeta}_{2} U_{2}\right)
$$

Note that the form of $\mathcal{L}_{T}$ violates the "Yukawa coupling" rule of the previous section, and there will indeed be 1-loop quadratic divergences in this theory. However, it is easy to see that these quadratic divergences do not generate masses for the little Higgses; instead they 
generate the plaquette interaction of the previous section! This is easy to see both from powercounting, and also directly from the quadratically divergent part of the Coleman-Weinberg potential, which is proportional to

$$
\frac{1}{16 \pi^{2}} \Lambda^{2} f^{2} \operatorname{tr}\left(\left|W_{1}\right|^{2}+\left|W_{2}\right|^{2}\right) \sim \text { const }+f^{4} \lambda_{1}^{*} \tilde{\lambda}_{1} \operatorname{tr} X_{1} X_{2}^{\dagger} X_{3} X_{4}^{\dagger}+f^{4} \lambda_{2}^{*} \tilde{\lambda}_{2} \operatorname{tr} X_{1} X_{4}^{\dagger} X_{3} X_{2}^{\dagger}
$$

Therefore the presence of the Yukawa interactions requires plaquette interactions with a natural size $\sim \lambda^{2}$. It is easy to extend our power-counting analysis to conclude that quadratically divergence little Higgs masses are only generated at 2-loop order as before. In particular, the operators

$$
\epsilon f^{4} \operatorname{tr} \mathbf{T}_{8} X_{1} X_{2}^{\dagger} X_{3} X_{4}^{\dagger}+\epsilon^{\prime} f^{4} \operatorname{tr} \mathbf{T}_{8} X_{1} X_{4}^{\dagger} X_{3} X_{2}^{\dagger}
$$

are generated; since they require $S U(3)$ breaking they have a natural size $\sim \lambda^{2} g^{2} /\left(16 \pi^{2}\right)$ or $\sim \lambda^{2} \zeta^{2} /\left(16 \pi^{2}\right)$, and will in general have non-zero imaginary parts. Therefore we also generate the $\epsilon$ plaquettes needed in the previous section, with the correct natural size! Due to the presence of these terms, all flat directions can be lifted an electroweak symmetry breaking can be triggered and stabilized. It is also straightforward to check that that any tadpole for the $\eta$ 's can only arise at 3-loop order or higher, and is sufficiently small.

After mixing with the Standard Model fermions, at low energies we have two massless Weyl fermions $q_{3}, u_{3}^{c}$ with a Yukawa couplings to a linear combination of little Higgses

$$
q_{3}\left(\alpha h_{x}+\beta h_{y}\right) u_{3}^{c}
$$

which give rise to top quark mass after electroweak symmetry breaking.

Note that the plaquette interactions have a natural size $\sim \lambda^{2}$ which is parametrically $\sim \lambda_{t}^{2}$. These give rise to a Higgs quartic potential which is $\sim \lambda_{t}^{2}$, and therefore in this model the physical Higgs mass is parametrically $m_{H} \sim m_{t}$.

\section{Conclusions}

In this paper we have presented a model of electroweak symmetry breaking accomplished by a naturally light Higgs scalar. The Higgs particle is a pseudo-Goldstone boson, and its mass is therefore protected against large radiative corrections. The technology of theory space is useful in constructing general models of this kind, eliminating sensitive dependence on short distance physics to arbitrary loop order. Since in constructing models of electroweak symmetry breaking this dependence need only be postponed to a scale of tens of $\mathrm{TeV}$, the extremely simple model presented here is entirely suitable as a realistic theory of electroweak symmetry breaking.

At the TeV scale the physics is perturbative and well described by an effective Lagrangian with a small number of parameters. There are only a small number of new states beyond the Standard Model. Counting all the helicity states, we introduce 56 new states beyond those of the Standard Model. As a point of comparison, the supersymmetric standard 
model introduces 126 new states. More importantly, the essential features of our model are characterized by very few parameters beyond the Standard Model. Electroweak symmetry breaking can be triggered through the top quark couplings, much as in the MSSM. There is some freedom in how the top quark couplings are incorporated, leading to the possibility that the entire Higgs potential can arise as a radiative effect at low energies. In this case the Higgs mass is naturally of the same size as the top quark mass.

In this effective theory, flavor changing interactions are only induced through the operators which give rise to the fermion Yukawa couplings, and dangerous flavor changing effects (often associated with physics beyond the standard model) do not arise. As always in an effective theory, there is the possibility of flavor changing neutral currents from physics above the cutoff, but it is straightforward to conceive scenarios where this also is not a problem.

For twenty years the domain of perturbative electroweak symmetry breaking models with a naturally light Higgs has been ruled by supersymmetric theories. In the last year a viable challenger has emerged: "little Higgs theories" which realize the Higgs as a pseudo-Goldstone boson in a low energy effective theory with a cutoff parametrically above the weak scale of order $\Lambda \sim 10 \mathrm{TeV}$. In this paper and its companion we have presented two minimal models of this sort. In the "minimal moose" model described here, two Higgs doublets, a complex weak triplet and a complex scalar are the only new degrees of freedom below the TeV scale. At the $\mathrm{TeV}$ scale a set of $S U(3)$ gauge bosons, an additional Higgs doublet, weak triplet and singlet and colored fermion appear. These same-statistics partners of the Standard Model fields are responsible for eliminating the 1-loop quadratic sensitivity of the little Higgs masses to the UV physics at scale $\Lambda$.

Our purpose in this paper and its companion has been to construct the smallest examples of the little Higgs phenomenon, both for the sake of economy as well as to illustrate the physics as clearly as possible. There is still much left to explore, and many further issues to be addressed by generalizations of these ideas. For example, perhaps the most compelling argument for low-energy supersymmetry is the spectacular prediction of the weak mixing angle with gauge coupling unification not far from the Planck scale. Recently it has been realized that the weak mixing angle can also be correctly predicted by electroweak unification into an $S U(3)$ symmetry at the $\sim 10 \mathrm{TeV}$ scale [19]. Little Higgs models offer the possibility to implement this mechanism in a natural way, and explicit models of this type are currently being constructed.

It is also of obvious interest to construct UV completions of these models at scales above $\Lambda$. The non-linear sigma model can be completed into a linear sigma model. This leads to a new "hierarchy problem" at the scale $10 \mathrm{TeV}$, which can then be solved by supersymmetry broken at $\sim 100 \mathrm{TeV}$, alleviating nearly all the conventional constraints on low-energy supersymmetry. More daringly, the linear sigma model fields themselves may arise as little Higgses in a larger theory, perhaps extrapolating to extremely high energies in a "cascade" of little Higgs models.

We can also imagine UV completions where the link fields emerge from fermion condensates in a strongly interacting gauge theory with a strong scale $\sim \Lambda \sim 10 \mathrm{TeV}$. Since we 
have seen that all the interactions required to produce the Higgs potential can be triggered from the same couplings generating the top quark mass, the main (and familiar) challenge is the implementation of the ETC-like interactions needed to generate the Yukawa coupling operators. However, the usual fatal flaws of strong dynamics at the $\mathrm{TeV}$ scale, such as large corrections to precision electroweak observables and too-light pseudo-Goldstone bosons, are eliminated in this framework, since the scale of strong dynamics would be well above the TeV scale. As we have seen the physics of electroweak symmetry breaking is still perturbative with a light Higgs, and so precision electroweak corrections are under control, and the lightest pseudo-Goldstone bosons are the little Higgses themselves. Furthermore, the difficulty of generating flavor without excessively large FCNCs is also greatly ameliorated [17], and is a more tractable model-building task.

The models presented here are new, fully realistic theories of $\mathrm{TeV}$ physics with natural electroweak symmetry breaking. As such the detailed phenomenology, constraints from precision low-energy measurements, and the implications for present and future colliders demand further exploration.

\section{Acknowledgments}

We would like to thank Sekhar Chivukula, Ken Lane and Martin Schmaltz for stimulating conversations. The work of A. Nelson and E. Katz was partially supported by the DOE under contract DE-FGO3-96-ER40956. N.A-H., T. Gregoire and J. Wacker are supported in part by the Department of Energy under Contracts DE-AC03-76SF00098 and the National Science Foundation under grant PHY-95-14797. A.G.C. is supported in part by the Department of Energy under grant number DE-FG02-91ER-40676. N.A.-H. is also supported by the Alfred P. Sloan foundation, and the David and Lucille Packard Foundation. T. Gregoire is also supported by an NSERC fellowship.

\section{References}

[1] N. Arkani-Hamed, A. G. Cohen, and H. Georgi, Electroweak symmetry breaking from dimensional deconstruction, Phys. Lett. B513 (2001) 232-240, [http://arXiv.org/abs/hep-ph/0105239].

[2] N. Arkani-Hamed, A. G. Cohen, T. Gregoire, and J. G. Wacker, Phenomenology of electroweak symmetry breaking from theory space, http://arXiv.org/abs/hep-ph/0202089.

[3] N. Arkani-Hamed, A. G. Cohen, and H. Georgi, (de)constructing dimensions, Phys. Rev. Lett. 86 (2001) 4757-4761, [http://arXiv.org/abs/hep-th/0104005].

[4] C. T. Hill, S. Pokorski, and J. Wang, Gauge invariant effective lagrangian for kaluza-klein modes, Phys. Rev. D64 (2001) 105005, [http://arXiv.org/abs/hep-th/0104035].

[5] N. Arkani-Hamed, A. G. Cohen, and H. Georgi, Twisted supersymmetry and the topology of theory space, http://arXiv.org/abs/hep-th/0109082.

[6] H. Georgi, A tool kit for builders of composite models, Nucl. Phys. B266 (1986) 274. 
[7] M. R. Douglas and G. Moore, D-branes, quivers, and ale instantons, hep-th/9603167.

[8] T. Gregoire and J. Wacker, Mooses, topology and higgs, http://arXiv.org/abs/hep-ph/0206023

[9] N. Arkani-Hamed, A. G. Cohen, E. Katz, and A. E. Nelson, The littlest higgs, http://arXiv.org/abs/hep-ph/0206021

[10] K. Lane, A case study in dimensional deconstruction, http://arXiv.org/abs/hep-ph/0202093.

[11] C. D. Froggatt and H. B. Nielsen, Hierarchy of quark masses, cabibbo angles and cp violation, Nucl. Phys. B147 (1979) 277.

[12] B. A. Dobrescu and C. T. Hill, Electroweak symmetry breaking via top condensation seesaw, Phys. Rev. Lett. 81 (1998) 2634-2637, [http://arXiv.org/abs/hep-ph/9712319].

[13] R. S. Chivukula, B. A. Dobrescu, H. Georgi, and C. T. Hill, Top quark seesaw theory of electroweak symmetry breaking, Phys. Rev. D59 (1999) 075003, [http://arXiv.org/abs/hep-ph/9809470].

[14] A. Manohar and H. Georgi, Chiral quarks and the nonrelativistic quark model, Nucl. Phys. B234 (1984) 189.

[15] A. G. Cohen, D. B. Kaplan, and A. E. Nelson, Counting 4pi's in strongly coupled supersymmetry, Phys. Lett. B412 (1997) 301-308, [http://arXiv.org/abs/hep-ph/9706275].

[16] M. A. Luty, Naive dimensional analysis and supersymmetry, Phys. Rev. D57 (1998) 1531-1538, [http://arXiv.org/abs/hep-ph/9706235].

[17] R. S. Chivukula, N. Evans, and E. H. Simmons, Flavor physics and fine-tuning in theory space, http://arXiv.org/abs/hep-ph/0204193.

[18] E. Katz and A. E. Nelson, in preparation, .

[19] S. Dimopoulos and D. E. Kaplan, The weak mixing angle from an su(3) symmetry at a tev, http://arXiv.org/abs/hep-ph/0201148. 